\title{
The reliability of local error estimators for convection-diffusion equations.
}

\author{
David Kay $^{\mathrm{a}} \quad$ David Silvester ${ }^{\mathrm{b}}$ \\ ${ }^{a}$ School of Mathematical Sciences, University of Sussex, Brighton BN1 9QH \\ (dkay@sussex.ac.uk) \\ ${ }^{b}$ Department of Mathematics, UMIST, Manchester M60 1QD \\ (na.silvester@na-net.ornl.gov). Research supported by EPSRC grants GR/K91262 \\ \& GR/L05617.
}

Oxford University Computing Laboratory

Numerical Analysis Group

Wolfson Building

Parks Road

Oxford, England OX1 3QD

November, 2000 


\begin{abstract}
We assess the reliability of a simple a posteriori error estimator for steady state convection-diffusion equations in cases where convection dominates. Our estimator is computed by solving a local Poisson problem with Neumann boundary conditions. It gives global upper and local lower bounds on the error measured in the $H^{1}$ semi-norm, except that the error may be over-estimated locally within boundary layers if these are not resolved by the mesh, that is, when the local mesh Péclet number is significantly greater than unity. We discuss the implications of this over-estimation in a practical context where the estimator is used as a local error indicator within a self-adaptive mesh refinement process.
\end{abstract}

\title{
1 Introduction
}

During the last two decades there has been a rapid development in practical a posteriori error estimation techniques for elliptic PDEs. This explosion in interest has been driven by the underlying need to increase the reliability and efficiency of finite element software for solving such problems, see Verfürth [15] for a general review. Convection-diffusion problems have local features which make them ripe for adaptive refinement algorithms based on local error indicators, although there remain some outstanding issues; firstly, the role of stabilisation e.g. using streamline upwind discretisations, and secondly, the fact that local error estimation may be very inaccurate if critical features like boundary layers are not adequately resolved by the underlying mesh, see Verfürth [14].

The performance of a simple error estimator in the presence of layers is the subject of this work. An outline of the paper is as follows. In the next section we define our local error estimator, and we establish theoretical bounds on its effectiveness that illustrates a potential source of difficulty, namely, boundary layers that are not resolved. Note that our estimator is reliable in the sense that (up to a constant factor) it provides a global upper bound on the error - the problem is that the estimator may provide a gross over-estimate. A set of numerical experiments is presented in $\S 3$ which highlights this deficiency. More promising results that are representative of performance when using the estimator in an adaptive refinement setting are described in $\S 4$. These latter results suggest that simple estimators can nevertheless provide a very effective refinement indicator if used in conjunction with streamline diffusion stabilisation. 


\section{Analysis of the local error estimator}

Given $\Omega$, a bounded polygonal domain in $\mathbb{R}^{2}$, with boundary $\partial \Omega=\bar{\Gamma}_{N} \cup \bar{\Gamma}_{D}, \Gamma_{N} \cap \Gamma_{D}=\emptyset$, our aim is to solve the following convection-diffusion problem: find $u$ such that

$$
\begin{array}{r}
-\epsilon \Delta u+\mathbf{b} \cdot \nabla u=f \text { in } \Omega \\
u=g \text { on } \Gamma_{D} \\
\frac{\partial u}{\partial n}=0 \text { on } \Gamma_{N},
\end{array}
$$

where $\epsilon>0$ is a viscosity parameter, and the convective wind $\mathbf{b}$ satisfies the following,

(A.1) $\quad \nabla \cdot \mathbf{b}=0$

(A.2) $\quad\|\mathbf{b}\|_{\infty, \Omega}=1$,

(A.3) $\quad \mathbf{b} \cdot \mathbf{n} \geq 0$ on $\Gamma_{N}$.

Using standard notation (see e.g. Braess [7, pp. 28-33]), the underlying bilinear form is

$$
B_{\epsilon}(u, v)=\epsilon(\nabla u, \nabla v)+(\mathbf{b} \cdot \nabla u, v),
$$

and is defined for all $u, v \in H^{1}(\Omega)$. The solution space is

$$
H_{g}^{1}(\Omega)=\left\{v \in H^{1}(\Omega): v=g \text { on } \Gamma_{D}\right\}
$$

and the variational problem associated with (2.1) is given by, find $u \in H_{g}^{1}(\Omega)$ such that

$$
B_{\epsilon}(u, v)=(f, v) \quad \forall v \in H_{0}^{1}(\Omega)
$$

To define a discrete version of (2.4) we require the following definitions:

1. $\mathcal{T}_{h}$ is a partition of $\Omega$ into triangles or convex quadrilaterals such that $\bar{T}_{1} \cap \bar{T}_{2}$ is either empty, a common edge, or a common vertex of $T_{1}$ and $T_{2}$ for all $T_{1}, T_{2} \in \mathcal{T}_{h}$,

2. $h_{T}:=\inf \{\operatorname{diam} S: S$ is a ball such that $T \subset S\}, T \in \mathcal{T}_{h}$,

3. $\rho_{T}:=\sup \{\operatorname{diam} S: S$ is a ball contained in $T\}, T \in \mathcal{T}_{h}$,

4. $\theta_{T}=h_{T} / \rho_{T}, \forall T \in \mathcal{T}_{h}$. Furthermore, for any domain $\Omega^{*} \subset \Omega$ consisting of a union of $T \in \mathcal{T}_{h}$, we let $\theta_{\Omega^{*}}$ denote the minimum $\theta_{T}, T \subset \Omega^{*}$. 
For simplicity, we restrict our attention to the lowest order linear triangular and bilinear rectangular elements (the extension to isoparametrically mapped quadrilaterals, and to the lowest order conforming three-dimensional approximation methods is immediate). Thus, for a partition $\mathcal{T}_{h}$ into triangular elements, we define

$$
X_{h}:=\left\{v \in H^{1}(\Omega):\left.v\right|_{T} \in \mathbb{P}^{1}(T) \quad \forall T \in \mathcal{T}_{h}\right\}
$$

whereas for a grid of rectangles we define

$$
X_{h}:=\left\{v \in H^{1}(\Omega):\left.v\right|_{T} \in \mathbb{Q}^{1}(T) \quad \forall T \in \mathcal{T}_{h}\right\}
$$

where $\mathbb{P}^{1}(T)$ is the space of polynomials of degree at most 1 on $T$, and $\mathbb{Q}^{1}(T)$ is the space of polynomials of complete degree 1 on $T$. Note that grids containing a mixture of triangles and rectangles are also possible.

\subsection{The streamline diffusion finite element approximation}

It is well known that the standard Galerkin approximation of (2.4) may produce an unstable, possibly oscillating, solution if the mesh is too coarse in critical regions. In such cases a posteriori error estimation in an adaptive refinement framework is relatively meaningless. To give additional stability on coarse meshes the streamline-diffusion finite element method will be used, see e.g. Johnson et al.[11] (see also the comments following the theorem in the next section). We will assume that the boundary data $g$ is continuous piecewise linear (our theoretical results extend in an obvious way when this is not the case).

The discrete (streamline-diffusion) approximation of (2.4) is to find $u_{h} \in X_{h}$, so that $\left.u_{h}\right|_{\Gamma_{D}}=g$ and

$$
\begin{aligned}
\epsilon\left(\nabla u_{h}, \nabla v\right)+\left(\mathbf{b} \cdot \nabla u_{h}, v\right)+\sum_{T \in \mathcal{T}_{h}} \delta_{T}\left(\mathbf{b} \cdot \nabla u_{h}, \mathbf{b} \cdot \nabla v\right)_{T}= \\
(f, v)+\sum_{T \in \mathcal{T}_{h}} \delta_{T}(f, \mathbf{b} \cdot \nabla v)_{T},
\end{aligned}
$$

for all $v \in X_{h}, v=0$ on $\Gamma_{D}$, see [11], where we have used the fact that $\left.\Delta v_{h}\right|_{T}=0$ for all $v_{h} \in X_{h}$. In (2.5) the local mesh Péclet number $P_{T}^{e}=\|\mathbf{b}\|_{\infty, T} h_{T} / \epsilon$ determines the streamline-diffusion coefficient $\delta_{T}$ in a given triangle $T \in \mathcal{T}_{h}$ via the "optimal" formula

$$
\delta_{T}=\left\{\begin{array}{l}
\frac{1}{2} h_{T}\left(1-\frac{1}{P_{T}^{e}}\right) \quad \text { if } P_{T}^{e}>1 \\
0 \text { if } P_{T}^{e} \leq 1
\end{array}\right.
$$


respectively, where $h_{T}$ is a measure of the element length in the direction of the wind. See Fischer et al. [9] for justification. An attractive feature of the streamline-diffusion approach is it leads to an a priori convergence estimate which is independent of $\epsilon$ in the streamline derivative $\mathbf{b} \cdot \nabla$, see $[11]$.

\subsection{Definition of the local error estimator}

Let $\mathcal{E}(T)$ denote the set of edges of $T$, and $\mathcal{E}_{h}=\cup_{T \in \mathcal{T}_{h}} \mathcal{E}(T)$ denote the set of all edges split into interior and boundary edges via

$$
\mathcal{E}_{h}:=\mathcal{E}_{h, \Omega} \cup \mathcal{E}_{h, D} \cup \mathcal{E}_{h, N}
$$

so that $\mathcal{E}_{h, \Omega}:=\left\{E \in \mathcal{E}_{h}: E \subset \Omega\right\}, \mathcal{E}_{h, D}:=\left\{E \in \mathcal{E}_{h}: E \subset \Gamma_{D}\right\}$ and $\mathcal{E}_{h, N}:=\{E \in$ $\left.\mathcal{E}_{h}: E \subset \Gamma_{N}\right\}$. For a given partition $\mathcal{T}_{h}$, we want to compute an approximation to the solution of the convection-diffusion problem (2.4), by solving the stabilised problem (2.5) with a stabilisation parameter $\delta_{T}$ satisfying (2.6). Then, denoting $e_{h}=u-u_{h}$, we seek a simple approximation to the error $e_{h}$ so that efficient refinement of the mesh can be made at each level of an adaptive solution process.

Since our intention is to apply our adaptive methodology within the field of incompressible flow modeling, we will keep away from the hyperbolic limit, and simply estimate the error in the $H^{1}$-seminorm. To this end, we modify the well-known Bank and Weiser estimator [6], and using an idea of Ainsworth and Oden [4], solve a local (element) Poisson problem, over a suitably chosen (higher-order) approximation space.

The data for the local problems is given by interior residuals and inter-element flux jumps. In particular, for any edge $E$ of an element $T \in \mathcal{T}_{h}$, we define the flux jump in the usual way via

$$
R_{E}= \begin{cases}\llbracket\left[\frac{\partial u_{h}}{\partial \mathbf{n}_{E}}\right]_{E} & E \in \mathcal{E}_{h, \Omega} \\ -2\left(\frac{\partial u_{h}}{\partial \mathbf{n}_{E}}\right) & E \in \mathcal{E}_{h, N} \\ 0 & E \in \mathcal{E}_{h, D}\end{cases}
$$

where $\frac{\partial u_{h}}{\partial \mathbf{n}_{E}}$ is a constant function on the inter-element edge $E$ and $\llbracket v \rrbracket_{E}$ measures the jump in $v$ across $E$, that is, for $E \in \mathcal{E}(T) \cap \mathcal{E}(S), T, S \in \mathcal{T}_{h}$ and defining $\mathbf{n}_{E, T}$ and $\mathbf{n}_{E, S}$ to be the outward normals with respect to the edge $E$ from elements $T$ and $S$ respectively, we have

$$
\llbracket v \rrbracket_{E}=\left.v\right|_{T} \mathbf{n}_{E, T}+\left.v\right|_{S} \mathbf{n}_{E, S}
$$


Since $\left.\Delta u_{h}\right|_{T}=0$ for all $T \in \mathcal{T}_{h}$, the element interior residual is given by the hyperbolic part of $(2.1)$

$$
R_{T}=\left.\left(f-\mathbf{b} \cdot \nabla u_{h}\right)\right|_{T}
$$

and we define

$$
R_{T}^{0}=\mathcal{P}_{T}^{0} R_{T},
$$

where $\mathcal{P}_{T}^{0}$ is taken here to be the $L^{2}(T)$ projection onto the space $\mathbb{P}^{0}(T)$, (see Verfürth [15, pp. 14]). For an element $T \in \mathcal{T}_{h}$, the estimator is given by

$$
\eta_{T}=\left\|\nabla e_{T}\right\|_{T}
$$

where $e_{T} \in \mathcal{Q}_{T}$ satisfies

$$
\epsilon\left(\nabla e_{T}, \nabla v\right)_{T}=\left(R_{T}^{0}, v\right)_{T}-\frac{1}{2} \epsilon \sum_{E \in \mathcal{E}(T)}<R_{E}, v>_{E},
$$

for all $v \in \mathcal{Q}_{T}$. Here $\mathcal{Q}_{T}$ is the "correction" space $\mathcal{Q}_{T}=Q_{T} \oplus B_{T}$ consisting of edge and interior bubble functions respectively;

$$
Q_{T}=\operatorname{span}\left\{\psi_{E}: E \in \mathcal{E}(T) \cap\left(\mathcal{E}_{h, \Omega} \cup \mathcal{E}_{h, N}\right)\right\}
$$

where $\psi_{E}: T \rightarrow \mathbb{R}$ is the usual quadratic (or bi-quadratic) edge-bubble which is zero on the other two edges of $T$ (see [15, pages $9-10]$ ). $B_{T}$ is the space spanned by interior cubic (or biquadratic) bubbles $\phi_{T}$ such that $0 \leq \phi_{T} \leq 1, \phi_{T}=1$ only at the centroid, and supp $\phi_{T} \subset T$. Note that the local problems are always well posed, and that for each triangular (or rectangular) element a $4 \times 4($ or $5 \times 5)$ problem must be solved to compute $e_{T}$.

Remark 1 In comparison with the related work of Verfürth [14] we note the following differences. Firstly, the PDE which is considered in [14] also allows control on the $L^{2}-$ norm of the error. Secondly, the local problems in [14] are discrete convection-diffusion problems of significantly larger dimension e.g. at least $12 \times 12$ in the triangular case.

Remark 2 The important issue of equilibration of the flux contribution is not addressed in this work, cf. the symmetric splitting in (2.7); see e.g. Babuška et al. [5], Ainsworth [2]. A technique for constructing equilibrated fluxes in the related case of singularly perturbed Helmholtz problems is analysed by Ainsworth and Babuška [3]. 


\subsection{Analysis}

The following analysis is an extension of the work of Verfürth. The notation is identical, see [15, page 9]; $\mathcal{N}_{T}$ is used to denote to be the set of vertices of $T \in \mathcal{T}_{h}$ and we define $\mathcal{N}_{h}=\cup_{T \in \mathcal{T}_{h}} \mathcal{N}_{T}$ to be the set of all vertices. We also define $\mathcal{N}_{E}$ be the set of vertices of $E \in \mathcal{E}_{h}$, and for $T \in \mathcal{T}_{h}, E \in \mathcal{E}_{h}$ and $\mathbf{x} \in \mathcal{N}_{h}$ we define local "patches" of elements via

$$
\begin{aligned}
\omega_{T} & =\cup_{\mathcal{E}(T) \cap \mathcal{E}\left(T^{\prime}\right) \neq \emptyset} T^{\prime} \\
\omega_{E} & =\cup_{E \in \mathcal{E}\left(T^{\prime}\right)} T^{\prime} \\
\tilde{\omega}_{T} & =\cup_{\mathcal{N}_{T} \cap \mathcal{N}_{T^{\prime}} \neq \emptyset} T^{\prime} \\
\tilde{\omega}_{E} & =\cup_{\mathcal{N}_{E} \cap \mathcal{N}_{T^{\prime}} \neq \emptyset} T^{\prime} .
\end{aligned}
$$

We now outline the proof of our main theoretical result, which shows that the estimator $\eta_{T}$ is equivalent to the true error away from boundary layers. In the following, we make frequent use of the short hand notation $\|f\|_{S}$ to denote the $L^{2}$ norm of a function in $L^{2}(S)$, and we use the letter $C$ to denote a constant which is independent of the parameters $h$ and $\epsilon$.

Theorem 1 For a given triangulation $\mathcal{T}_{h}$, the estimator $\eta_{T} \equiv\left\|\nabla e_{T}\right\|_{T}$ satisfies

$$
\left\|\nabla e_{h}\right\|_{\Omega} \leq C\left(\theta_{\Omega}\right)\left(\sum_{T \in \mathcal{T}_{h}} \eta_{T}^{2}+\sum_{T \in \mathcal{T}_{h}}\left(\frac{h_{T}}{\epsilon}\right)^{2}\left\|R_{T}-R_{T}^{0}\right\|_{T}^{2}\right)^{1 / 2} .
$$

Furthermore, for a given $T \in \mathcal{T}_{h}$

$$
\eta_{T} \leq C\left(\theta_{\omega_{T}}\right)\left(\left\|\nabla e_{h}\right\|_{\omega_{T}}+\sum_{T \subset \omega_{T}} \frac{h_{T}}{\epsilon}\left\|\mathbf{b} \cdot \nabla e_{h}\right\|_{T}+\sum_{T \subset \omega_{T}} \frac{h_{T}}{\epsilon}\left\|R_{T}-R_{T}^{0}\right\|_{T}\right) .
$$

Proof Because of assumptions (A.1)-(A.3) we have

$$
B_{\epsilon}\left(e_{h}, e_{h}\right) \geq \epsilon\left\|\nabla e_{h}\right\|_{\Omega}^{2} .
$$

Using Galerkin orthogonality (and (2.4) and (2.5)) we have that

$$
\begin{aligned}
B_{\epsilon}\left(e_{h}, v\right) & =B_{\epsilon}\left(e_{h}, v-v_{h}\right)+B_{\epsilon}\left(e_{h}, v_{h}\right) \\
& =B_{\epsilon}\left(e_{h}, v-v_{h}\right)-\sum_{T \in \mathcal{T}_{h}} \delta_{T}\left(f-\mathbf{b} \cdot \nabla u_{h}, \mathbf{b} \cdot \nabla v_{h}\right)_{T},
\end{aligned}
$$

for any $v \in H_{0}^{1}(\Omega)$ and $v_{h} \in X_{h} \cap H_{0}^{1}(\Omega)$. Furthermore, there exists an interpolant (see e.g. Clément [8]), $e_{h}^{*} \in X_{h} \cap H_{0}^{1}(\Omega)$ such that, for all $T \in \mathcal{T}_{h}$

$$
\left\|e_{h}-e_{h}^{*}\right\|_{T} \leq C_{1}\left(\theta_{\tilde{\omega}_{T}}\right) h_{T}\left\|\nabla e_{h}\right\|_{\tilde{\omega}_{T}},
$$


and for all $E \in \mathcal{E}_{h}$

$$
\left\|e_{h}-e_{h}^{*}\right\|_{E} \leq C_{2}\left(\theta_{\tilde{\omega}_{E}}\right) h_{E}^{1 / 2}\left\|\nabla e_{h}\right\|_{\tilde{\omega}_{E}} .
$$

Hence using (2.14), and choosing $v=e_{h}$ and $v_{h}=e_{h}^{*}$ in (2.15) gives

$$
\epsilon\left\|\nabla e_{h}\right\|_{\Omega}^{2} \leq\left(f, e_{h}-e_{h}^{*}\right)-B_{\epsilon}\left(u_{h}, e_{h}-e_{h}^{*}\right)-\sum_{T \in \mathcal{T}_{h}} \delta_{T}\left(f-\mathbf{b} \cdot \nabla u_{h}, \mathbf{b} \cdot \nabla e_{h}^{*}\right)_{T} .
$$

Integrating by parts over each element, and noting that $e_{h}=e_{h}^{*}$ on $\Gamma_{D}$ then gives

$$
\begin{aligned}
& \epsilon\left\|\nabla e_{h}\right\|_{\Omega}^{2} \leq \sum_{T \in \mathcal{T}_{h}}\left[\left(f-\mathbf{b} \cdot \nabla u_{h}, e_{h}-e_{h}^{*}\right)_{T}-\delta_{T}\left(f-\mathbf{b} \cdot \nabla u_{h}, \mathbf{b} \cdot \nabla e_{h}^{*}\right)_{T}\right. \\
& \left.+\frac{1}{2} \epsilon \sum_{E \in \mathcal{E}(T) \cap \mathcal{E}_{h, \Omega}}\left\langle\llbracket \frac{\partial u_{h}}{\partial \mathbf{n}_{E}} \rrbracket_{E}, e_{h}-e_{h}^{*}\right\rangle_{E}-\epsilon \sum_{E \in \mathcal{E}(T) \cap \mathcal{E}_{h, N}}\left\langle\frac{\partial u_{h}}{\partial \mathbf{n}_{E}}, e_{h}-e_{h}^{*}\right\rangle_{E}\right],
\end{aligned}
$$

and from the definition of $R_{T}^{0}$ and $R_{E}$ we have

$$
\begin{aligned}
\epsilon\left\|\nabla e_{h}\right\|_{\Omega}^{2} \leq \sum_{T \in \mathcal{T}_{h}} & {\left[\left(R_{T}^{0}, e_{h}-e_{h}^{*}\right)_{T}+\left(R_{T}-R_{T}^{0}, e_{h}-e_{h}^{*}\right)_{T}-\delta_{T}\left(R_{T}^{0}, \mathbf{b} \cdot \nabla e_{h}^{*}\right)_{T}\right.} \\
& \left.-\delta_{T}\left(R_{T}-R_{T}^{0}, \mathbf{b} \cdot \nabla e_{h}^{*}\right)_{T}+\frac{1}{2} \epsilon \sum_{E \in \mathcal{E}(T)}\left\langle R_{E}, e_{h}-e_{h}^{*}\right\rangle_{E}\right] .
\end{aligned}
$$

Then, since $\delta_{T} \leq h_{T}$ (from (2.6)), using the obvious bound $\left\|\mathbf{b} \cdot \nabla e_{h}^{*}\right\|_{T} \leq 2\left\|\nabla e_{h}\right\|_{T}$ together with the interpolation bounds gives

$$
\begin{gathered}
\epsilon\left\|\nabla e_{h}\right\|_{\Omega}^{2} \leq C\left(\theta_{\Omega}\right) \sum_{T \in \mathcal{T}_{h}}\left[h_{T}\left\|R_{T}^{0}\right\|_{T}\left\|\nabla e_{h}\right\|_{\tilde{\omega}_{T}}+h_{T}\left\|R_{T}-R_{T}^{0}\right\|_{T}\left\|\nabla e_{h}\right\|_{\tilde{\omega}_{T}}\right. \\
\left.+\frac{1}{2} \epsilon \sum_{E \in \mathcal{E}(T)} h_{E}^{1 / 2}\left\|R_{E}\right\|_{E}\left\|\nabla e_{h}\right\|_{\tilde{\omega}_{E}}\right]
\end{gathered}
$$

which can be bounded via a discrete Cauchy-Schwarz inequality to give the bound

$$
\begin{gathered}
\epsilon\left\|\nabla e_{h}\right\|_{\Omega}^{2} \leq C\left(\theta_{\Omega}\right)\left(\sum_{T \in \mathcal{T}_{h}}\left\|\nabla e_{h}\right\|_{\tilde{\omega}_{T}}^{2}\right)^{1 / 2}\left(\sum _ { T \in \mathcal { T } _ { h } } \left[h_{T}^{2}\left\|R_{T}^{0}\right\|_{T}^{2}+h_{T}^{2}\left\|R_{T}-R_{T}^{0}\right\|_{T}^{2}\right.\right. \\
\left.\left.+\sum_{E \in \mathcal{E}(T) \cap \mathcal{E}_{h, \Omega}} \frac{1}{4} h_{E} \epsilon^{2}\left\|R_{E}\right\|_{E}^{2}\right]\right)^{1 / 2} .
\end{gathered}
$$

The rest of the proof is a generalisation of the standard argument, see e.g. Verfürth $[15$, pp. 15-18]. For $T \in \mathcal{T}_{h}$, we define $w_{T}=R_{T}^{0} \phi_{T}$. Then since $R_{T}^{0} \in \mathbb{P}^{0}(T)$ and $\phi_{T} \leq 1$, it is easy to show that

$$
\left\|R_{T}^{0}\right\|_{T}^{2} \leq C\left(R_{T}^{0}, w_{T}\right)_{T}
$$




$$
\left\|w_{T}\right\|_{T} \leq\left\|R_{T}^{0}\right\|_{T}
$$

Using (2.7) with (2.17), (2.18) and the inverse estimate $\left\|\nabla w_{T}\right\|_{T} \leq C h_{T}^{-1}\left\|w_{T}\right\|_{T}$, we have

$$
\begin{aligned}
\left\|R_{T}^{0}\right\|_{T}^{2} & \leq C\left(R_{T}^{0}, w_{T}\right)_{T}=C \epsilon\left(\nabla e_{T}, \nabla w_{T}\right)_{T} \\
& \leq C \epsilon\left\|\nabla e_{T}\right\|_{T}\left\|\nabla w_{T}\right\|_{T} \\
& \leq C h_{T}^{-1} \epsilon\left\|\nabla e_{T}\right\|_{T}\left\|R_{T}^{0}\right\|_{T}
\end{aligned}
$$

Similarly, for $E \in \mathcal{E}(T)$, we define $w_{E}=R_{E} \psi_{E} \in \mathcal{Q}_{T}$. Using (2.7), and then combining the minimum angle condition with standard scaling results (see e.g. Kay \& Silvester [12, Lemma 3.2]), we have

$$
\begin{aligned}
\epsilon\left\|R_{E}\right\|_{E}^{2} & \leq C \epsilon<R_{E}, w_{E}>_{E}=C \sum_{T^{\prime} \subset \omega_{E}}\left[-\epsilon\left(\nabla e_{T^{\prime}}, \nabla w_{E}\right)_{T^{\prime}}+\left(R_{T^{\prime}}^{0}, w_{E}\right)_{T^{\prime}}\right] \\
& \leq C \sum_{T^{\prime} \subset \omega_{E}}\left[\epsilon\left\|\nabla e_{T^{\prime}}\right\|_{T^{\prime}}\left\|\nabla w_{E}\right\|_{T^{\prime}}+\left\|R_{T^{\prime}}^{0}\right\|_{T^{\prime}}\left\|w_{E}\right\|_{T^{\prime}}\right] \\
& \leq C\left(\theta_{\omega_{E}}\right)\left\|R_{E}\right\|_{E} \sum_{T^{\prime} \subset \omega_{E}}\left[\epsilon\left\|\nabla e_{T^{\prime}}\right\|_{T^{\prime}} h_{E}^{-1 / 2}+\left\|R_{T^{\prime}}^{0}\right\|_{T^{\prime}} h_{T^{\prime}}^{1 / 2}\right] .
\end{aligned}
$$

Finally, combining (2.16), (2.19) and (2.20) gives the required global upper bound (2.12).

We now show the local lower bound (2.13). Starting from the local problem (2.7) and choosing $v=e_{T}$, we have

$$
\begin{aligned}
\epsilon\left\|\nabla e_{T}\right\|_{T}^{2} & =\left(R_{T}^{0}, e_{T}\right)_{T}-\frac{1}{2} \epsilon \sum_{E \in \mathcal{E}(T)}<R_{E}, e_{T}>_{E} \\
& \leq\left\|R_{T}^{0}\right\|_{T}\left\|e_{T}\right\|_{T}+\frac{1}{2} \epsilon \sum_{E \in \mathcal{E}(T)}\left\|R_{E}\right\|_{E}\left\|e_{T}\right\|_{E} .
\end{aligned}
$$

Since $e_{T} \in \mathcal{Q}_{T}$, a scaling argument gives

$$
\begin{aligned}
\left\|e_{T}\right\|_{T} & \leq C\left(\theta_{T}\right) h_{T}\left\|\nabla e_{T}\right\|_{T} \\
\left\|e_{T}\right\|_{E} & \leq C\left(\theta_{T}\right) h_{E}^{1 / 2}\left\|\nabla e_{T}\right\|_{T} .
\end{aligned}
$$

hence

$$
\epsilon\left\|\nabla e_{T}\right\|_{T} \leq h_{T}\left\|R_{T}^{0}\right\|_{T}+\frac{1}{2} \epsilon \sum_{E \in \mathcal{E}(T)} h_{E}^{1 / 2}\left\|R_{E}\right\|_{E} .
$$

Thus to get (2.13), we need to bound $h_{T}\left\|R_{T}^{0}\right\|_{T}$ and $h_{E}^{1 / 2}\left\|R_{E}\right\|_{E}$. For $T \in \mathcal{T}_{h}$, define $w_{T}$ as above. Then,

$$
C\left\|R_{T}^{0}\right\|_{T}^{2} \leq\left(R_{T}^{0}, w_{T}\right)_{T}
$$




$$
\begin{aligned}
& =\left(R_{T}^{0}-R_{T}, w_{T}\right)+B_{\epsilon}\left(u, w_{T}\right)-\left(\mathbf{b} \cdot \nabla u_{h}, w_{T}\right)_{T} \\
& =\left(R_{T}^{0}-R_{T}, w_{T}\right)+B_{\epsilon}\left(u, w_{T}\right)-B_{\epsilon}\left(u_{h}, w_{T}\right) \\
& =\left(R_{T}^{0}-R_{T}, w_{T}\right)_{T}+\epsilon\left(\nabla e_{h}, \nabla w_{T}\right)_{T}+\left(\mathbf{b} \cdot \nabla e_{h}, w_{T}\right)_{T} \\
& \leq\left\|R_{T}^{0}-R_{T}\right\|_{T}\left\|w_{T}\right\|_{T}+\epsilon\left\|\nabla e_{h}\right\|_{T}\left\|\nabla w_{T}\right\|_{T}+\left\|\mathbf{b} \cdot \nabla e_{h}\right\|_{T}\left\|w_{T}\right\|_{T} .
\end{aligned}
$$

Hence, using (2.18) and the inverse estimate

$$
h_{T}\left\|R_{T}^{0}\right\|_{T} \leq C\left(h_{T}\left\|R_{T}^{0}-R_{T}\right\|_{T}+\epsilon\left\|\nabla e_{h}\right\|_{T}+h_{T}\left\|\mathbf{b} \cdot \nabla e_{h}\right\|_{T}\right) .
$$

For $E \in \mathcal{E}(T) \cap \mathcal{E}_{h, \Omega}$ we use $w_{E}$, and proceed in a similar way

$$
\begin{aligned}
C \epsilon\left\|R_{E}\right\|_{E}^{2} \leq & \epsilon<R_{E}, w_{E}>_{E}=\sum_{T^{\prime} \subset \omega_{E}} \epsilon\left(\nabla u_{h}, \nabla w_{E}\right)_{T^{\prime}} \\
& =\sum_{T^{\prime} \subset \omega_{E}}\left[\left(R_{T^{\prime}}-R_{T^{\prime}}^{0}, w_{E}\right)_{T^{\prime}}-\epsilon\left(\nabla e_{h}, \nabla w_{E}\right)_{T^{\prime}}\right. \\
& \left.\quad-\left(\mathbf{b} \cdot \nabla e_{h}, w_{E}\right)_{T^{\prime}}+\left(R_{T^{\prime}}^{0}, w_{E}\right)_{T^{\prime}}\right] \\
& \sum_{T^{\prime} \subset \omega_{E}}\left[\left\|R_{T^{\prime}}^{0}-R_{T^{\prime}}\right\|_{T^{\prime}}\left\|w_{E}\right\|_{T^{\prime}}+\epsilon\left\|\nabla e_{h}\right\|_{T^{\prime}}\left\|\nabla w_{E}\right\|_{T^{\prime}}\right. \\
& \left.\quad+\left\|\mathbf{b} \cdot \nabla e_{h}\right\|_{T^{\prime}}\left\|w_{E}\right\|_{T^{\prime}}+\left\|R_{T^{\prime}}^{0}\right\|_{T^{\prime}}\left\|w_{E}\right\|_{T^{\prime}}\right] .
\end{aligned}
$$

Thus,

$$
\begin{array}{r}
\epsilon\left\|R_{E}\right\|_{E}^{2} \leq C\left\|R_{E}\right\|_{E} h_{E}^{-1 / 2} \sum_{T^{\prime} \subset \omega_{E}}\left[h_{T^{\prime}}\left\|R_{T^{\prime}}^{0}-R_{T^{\prime}}\right\|_{T^{\prime}}+\epsilon\left\|\nabla e_{h}\right\|_{T^{\prime}}\right. \\
\left.+h_{T^{\prime}}\left\|\mathbf{b} \cdot \nabla e_{h}\right\|_{T^{\prime}}+h_{T^{\prime}}\left\|R_{T^{\prime}}^{0}\right\|_{T^{\prime}}\right] .
\end{array}
$$

Using (2.23) to bound the last term, we have

$$
\epsilon h_{E}^{1 / 2}\left\|R_{E}\right\|_{E} \leq C \sum_{T^{\prime} \subset \omega_{E}}\left[h_{T^{\prime}}\left\|R_{T^{\prime}}^{0}-R_{T^{\prime}}\right\|_{T^{\prime}}+\epsilon\left\|\nabla e_{h}\right\|_{T^{\prime}}+h_{T^{\prime}}\left\|\mathbf{b} \cdot \nabla e_{h}\right\|_{T^{\prime}}\right]
$$

and then substituting (2.25) and (2.23) into (2.22) gives the stated local lower bound.

Remark 3 In the case of $\mathbb{P}_{1}$ triangles, if $f$ and $\mathbf{b}$ are piecewise constant functions then the consistency error term $\left\|R_{T}-R_{T}^{0}\right\|_{T}$ is identically zero, and we have a reliable estimator; i.e. the upper bound (2.12) is independent of $h$ and $\epsilon$.

In general, the consistency error represents a high order perturbation term (in particular, if $R_{T} \in H^{k}(T), k \in(0,1]$, then $\frac{h_{T}}{\epsilon}\left\|R_{T}-R_{T}^{0}\right\|_{T}$ may be bounded by $\left.\frac{h_{T}^{k+1}}{\epsilon}\left\|R_{T}\right\|_{k, T}\right)$. Note also that the size of the consistency error can be reduced by solving the local 
problems using higher order polynomial spaces, since higher degree approximations to $R_{T}$ can then be used, see [14]. Finally, checking through the proof of the theorem in the case $\delta_{T} \equiv 0$ it is clear that the same bounds, (2.12), (2.13), hold in the case of the standard Galerkin approximation method (but see the discussion in the next section).

\subsection{The effectivity of the estimator}

The lower bound (2.13) suggests that the local efficiency of the estimator could deteriorate in proportion to the mesh Péclet number in regions where $h_{T} \epsilon^{-1}\left\|\mathbf{b} \cdot \nabla e_{h}\right\|_{T}$ is comparable to $\left\|\nabla e_{h}\right\|_{T}$. In the case of small $\epsilon$, the important features of the solution $u$ are those of exponential boundary layers, and parabolic boundary and interior layers, see Roos et al. [13, chapter 3]. Furthermore, the asymptotic form of the solution in these cases implies that it is only layers of exponential boundary type that lead to the term $h_{T} \epsilon^{-1}\left\|\mathbf{b} \cdot \nabla e_{h}\right\|_{T}$ dominating the $H^{1}$-seminorm error. An important point here is that in the case of incompressible flow models based on the incompressible Navier-Stokes equations, exponential boundary layers only arise when outflow boundary conditions are inappropriately chosen, see e.g. Gresho and Sani [10].

Comparing the standard Galerkin and streamline diffusion methods, we have the a priori error estimates (for $u \in H^{k+1}(\Omega), k \in[0,1]$ )

$$
\begin{aligned}
\left\|\mathbf{b} \cdot \nabla e_{h}\right\|_{\Omega} & \leq C \frac{h^{k}}{\sqrt{\epsilon}}\|u\|_{k+1, \Omega}, \\
\left\|\mathbf{b} \cdot \nabla e_{h}\right\|_{\Omega} & \leq C h^{k}\|u\|_{k+1, \Omega}
\end{aligned}
$$

for the streamline derivative error, and

$$
\begin{aligned}
\left\|\mathbf{b}^{\perp} \cdot \nabla e_{h}\right\|_{\Omega} & \leq C \frac{h^{k}}{\sqrt{\epsilon}}\|u\|_{k+1, \Omega}, \\
\left\|\mathbf{b}^{\perp} \cdot \nabla e_{h}\right\|_{\Omega} & \leq C h^{k} \sqrt{\frac{h}{\epsilon}}\|u\|_{k+1, \Omega},
\end{aligned}
$$

for the cross wind error, respectively, see [11]. Hence, when $\epsilon<h$ a streamline diffusion method may improve the streamline derivative approximation by a factor $\sqrt{\epsilon} / h$ compared to the cross wind derivative, while no such improvement is seen for the standard Galerkin method. In this case we might expect to see an improved effectivity in the estimator when using streamline diffusion method-since the second term in the lower bound (2.13) is relatively $\sqrt{\epsilon / h}$ better than the first term. 
In the next section we present some computational results that demonstrate that, in the standard Galerkin case (i.e. $\delta_{T} \equiv 0$ in (2.5)), the bounds (2.13) and (2.12) are tight. Our numerical results also show that the effectiveness of the estimator is substantially improved if streamline up winding is applied. Specifically, if $\delta_{T}$ satisfies (2.6), and $\epsilon$ is reduced while keeping the other parameters fixed, then the ratio of the upper and lower bounds on the true error blows up like $\sqrt{P_{T}^{e}}$ instead of $P_{T}^{e}$.

Remark 4 The deterioration in efficiency above was also observed by Agarwal and Pinsky [1] in the context of a one-dimensional model problem. It is shown in [1] (at least in the one-dimensional case) that the effectivity index can be bounded independently of the mesh Péclet number if a local estimator is computed by solving a local convectiondiffusion problem with a judicious choice of local stabilisation parameter.

\section{$3 \quad$ Numerical experiments I: Effectivity indices}

In the light of our theoretical results, we define a test problem which contains an exponential boundary layer. One such problem corresponds to the case of a constant (vertical) wind $\mathbf{b}=(0,1)$, and zero forcing $f=0$, and the solution is given by

$$
u=x \frac{\left(1-e^{\frac{y-1}{\epsilon}}\right)}{\left(1-e^{-\frac{2}{\epsilon}}\right)} .
$$

We thus solve (2.5) using uniform $N \times N$ grids of square elements, i.e. $\mathbb{Q}^{1}$ approximation, over a domain $[0,1] \times[0,1]$, with interpolated data specified everywhere on the boundary (so that $\Gamma \equiv \Gamma_{D}$ ). To illustrate the importance of streamline-diffusion stabilisation when $P_{T}^{e}>1$, we consider the case $N=16, \epsilon=1 / 1024$, and plot isolines of the computed solution and the estimated errors (computed using (2.7)) in figures 1 and 2. Note that in the streamline-diffusion case the errors are (correctly) confined to the elements defining the boundary layer, whereas without stabilisation the errors propagate back along the approximate characteristics (potentially leading to refinement in the wrong place if used as an error indicator in an adaptive framework).

Given $u_{h}$ on a grid, we compute approximations to the exact errors on each element $\left\|\nabla e_{h}\right\|_{T}$ using adaptive Gaussian quadrature*. Defining the global error by

\footnotetext{
*Using quad2dg from the MATLAB Numerical Integration Toolbox.
} 

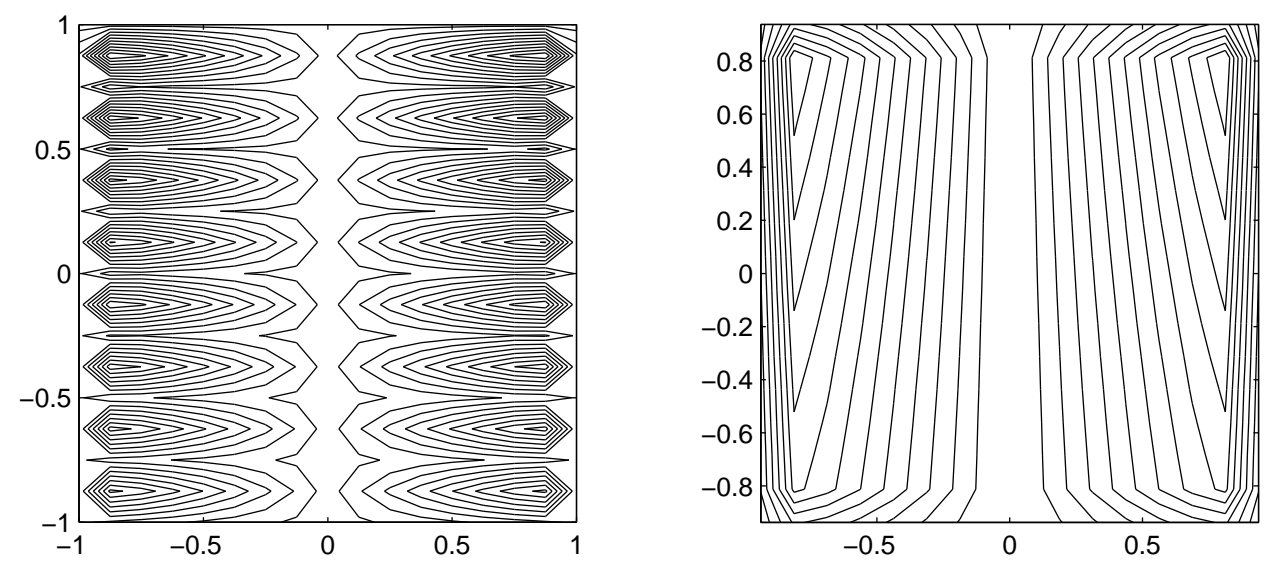

Figure 1: Galerkin solution and estimated errors: $16 \times 16$ uniform grid.
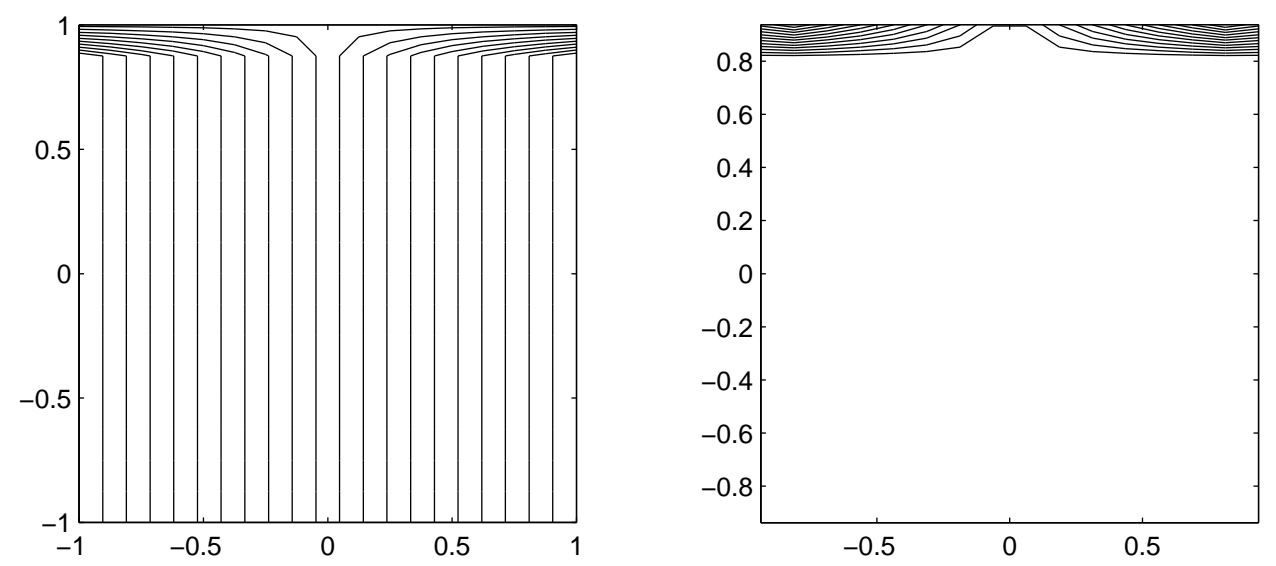

Figure 2: Streamline-Diffusion solution and estimated errors: $16 \times 16$ uniform grid. 
$\eta=\left(\sum_{T \in \mathcal{T}_{h}} \eta_{T}^{2}\right)^{1 / 2}$, the global effectivity index, $E_{\eta}:=\eta /\left\|\nabla e_{h}\right\|_{\Omega}$ can then be calculated. Some representative results computed in the unstabilised and stabilised cases are given in Table 1 and Table 2 respectively.

\begin{tabular}{|l|c|c|c|}
\hline$\epsilon$ & $\left\|\nabla e_{h}\right\|_{\Omega}$ & $\eta$ & $E_{\eta}$ \\
\hline $1 / 16$ & $1.850 \mathrm{e} 0$ & $3.026 \mathrm{e} 0$ & $3.22 \mathrm{e} 0$ \\
\hline $1 / 64$ & $5.616 \mathrm{e} 0$ & $3.217 \mathrm{e} 1$ & $5.73 \mathrm{e} 0$ \\
\hline $1 / 256$ & $1.652 \mathrm{e} 1$ & $4.388 \mathrm{e} 2$ & $2.65 \mathrm{e} 1$ \\
\hline $1 / 1024$ & $5.877 \mathrm{e} 1$ & $6.778 \mathrm{e} 3$ & $1.15 \mathrm{e} 2$ \\
\hline $1 / 4096$ & $2.274 \mathrm{e} 2$ & $1.075 \mathrm{e} 5$ & $4.73 \mathrm{e} 2$ \\
\hline
\end{tabular}

Table 1: Galerkin solution: exact and estimated errors on $8 \times 8$ uniform grid

\begin{tabular}{|l|c|c|c|}
\hline$\epsilon$ & $\left\|\nabla e_{h}\right\|_{\Omega}$ & $\eta$ & $E_{\eta}$ \\
\hline $1 / 16$ & $1.675 \mathrm{e} 0$ & $2.114 \mathrm{e} 0$ & $1.26 \mathrm{e} 0$ \\
\hline $1 / 64$ & $4.335 \mathrm{e} 0$ & $8.852 \mathrm{e} 0$ & $2.04 \mathrm{e} 0$ \\
\hline $1 / 256$ & $9.101 \mathrm{e} 0$ & $3.613 \mathrm{e} 1$ & $3.97 \mathrm{e} 0$ \\
\hline $1 / 1024$ & $1.841 \mathrm{e} 1$ & $1.454 \mathrm{e} 2$ & $7.90 \mathrm{e} 0$ \\
\hline
\end{tabular}

Table 2: Streamline-Diffusion solution: exact and estimated errors on $8 \times 8$ uniform grid

An important point that needs to be emphasised here is that the global effectivity index is always close to unity when $h$ is such that the mesh Péclet number is less than one (in which case there is no stabilisation), at least for the uniform grids considered here. If the grid is not sufficiently fine then the generic situation is as suggested by the results in Tables 1 and 2; viz, the effectivity index behaves like $P_{T}^{e}$ when $\epsilon \rightarrow 0$ for fixed $h$ if there is no stabilisation (as predicted by Theorem 2.1), and behaves like $\sqrt{P_{T}^{e}}$ otherwise. Effectivity indices computed using the $32 \times 32$ grid are plotted in figure 3, and in this case we see that streamline-diffusion stabilisation leads to $O(1)$ effectivity indices for all $\epsilon \geq O\left(10^{-3}\right)$. 


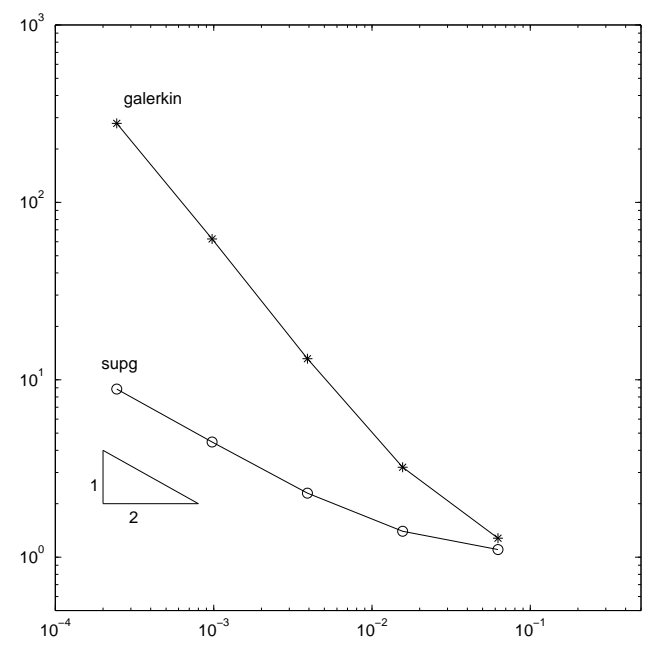

Figure 3: Effectivity indices vs $\epsilon: 32 \times 32$ uniform grid.

\section{Numerical experiments II: Adaptivity}

We conclude with an assessment of the effectiveness of the simple error estimator (2.7) when used as an error indicator within an adaptive refinement setting. We consider two examples; the first has an interior layer, and the second has both an interior and boundary layer. We use triangular elements, i.e. $\mathbb{P}^{1}$ approximation, and the streamline diffusion formulation (2.5) and (2.6) together with the following local refinement strategy.

1. For all $T \in \mathcal{T}_{n}$ obtain an error estimate $\eta_{T}$, and define $\eta^{*}=\max _{T \in \mathcal{T}_{n}} \eta_{T}$.

2. Define $\mathcal{T}_{n}^{*}=\left\{K: \eta_{K}>\alpha \eta^{*}\right\}$, for a specific choice of $\alpha \in(0,1)$.

3. Refine all elements $K \in \mathcal{T}_{n}^{*}$ into four similar elements. Remove hanging nodes (using longest edge bisection) to obtain $\mathcal{T}_{n+1}$.

\subsection{Interior Layers}

The following example shows that the error estimator $\eta$ is effective in locating and resolving interior layers. We solve (2.1) over a unit square domain with $\epsilon=1 / 6400$. The wind is given by $\mathbf{b}=\left(2 y\left(1-x^{2}\right),-2 x\left(1-y^{2}\right)\right)$ and forcing term $f$ is zero. There is a discontinuity in the inflow boundary $x=0$, viz

$$
u=\left\{\begin{array}{lc}
0 & 0 \leq y \leq 1 / 2 \\
\frac{1}{2 \sqrt{\epsilon}}(2 y-1) & 1 / 2 \leq y \leq 1 / 2+\sqrt{\epsilon} \\
1 & 1 / 2 \leq y \leq 1
\end{array}\right.
$$


and $u=1$ on the characteristic boundaries $y=1$ and $x=1$. The outflow boundary is

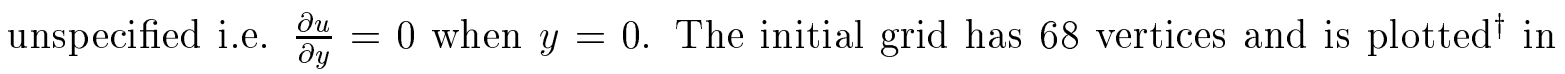
figure 4 , together with a contour plot of the computed solution. The computed solution and grid after 13 steps of adaptive refinement using a subdivision parameter $\alpha=0.5$ is plotted in figure 5. Note the excellent resolution of the interior layer.
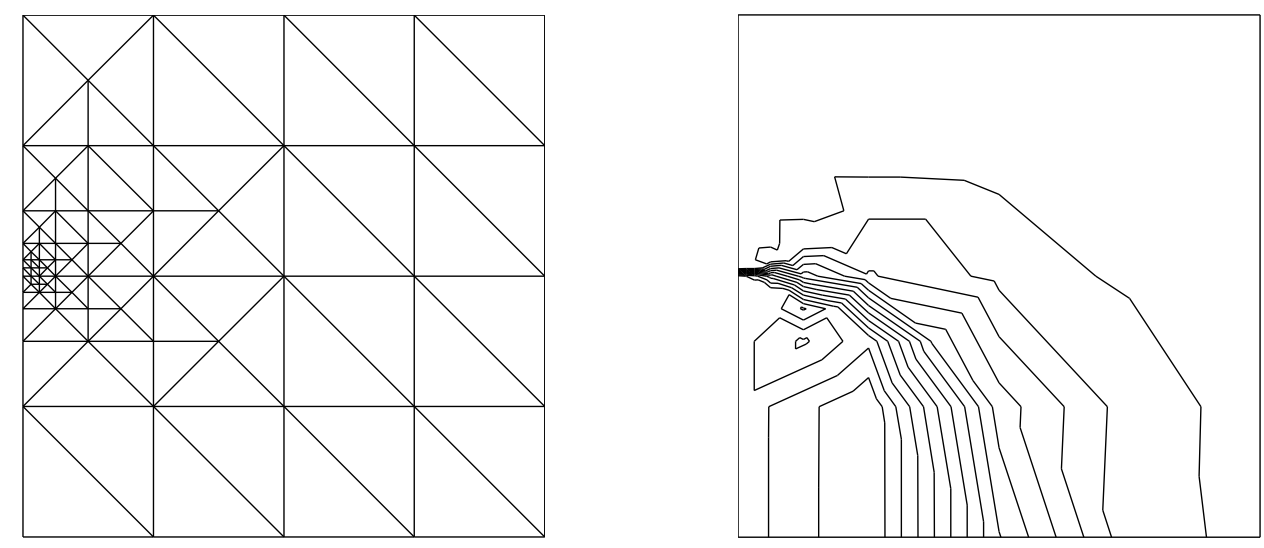

Figure 4: Initial grid and solution to 4.1: 68 dof.
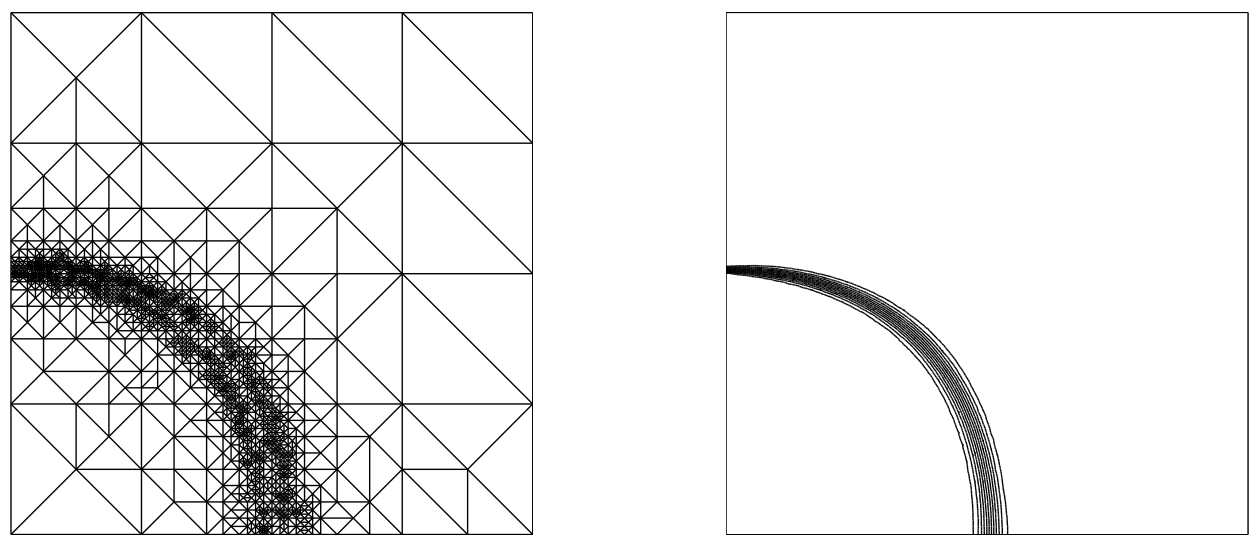

Figure 5: Adapted grid and solution to 4.1: 1572 dof.

\subsection{Boundary Layers}

Our final example is a more challenging test problem with both a boundary layer and an interior layer (see e.g. [9]). We solve (2.1) over a unit square domain with $\epsilon=1 / 6400$. The wind is given by $\mathbf{b}=(1,0)$ and the forcing term $f$ is zero. There is a discontinuity

\footnotetext{
${ }^{\dagger}$ Using the MATLAB PDE Toolbox.
} 
in the inflow boundary $x=0$ as above, and we specify the incompatible condition $u=0$ on the outflow boundary $x=1$. The characteristic boundaries are left unspecified i.e. $\frac{\partial u}{\partial y}=0$ when $y=0$ and $y=1$. The initial grid and a contour plot of the computed finite element solution is given in figure 6 .
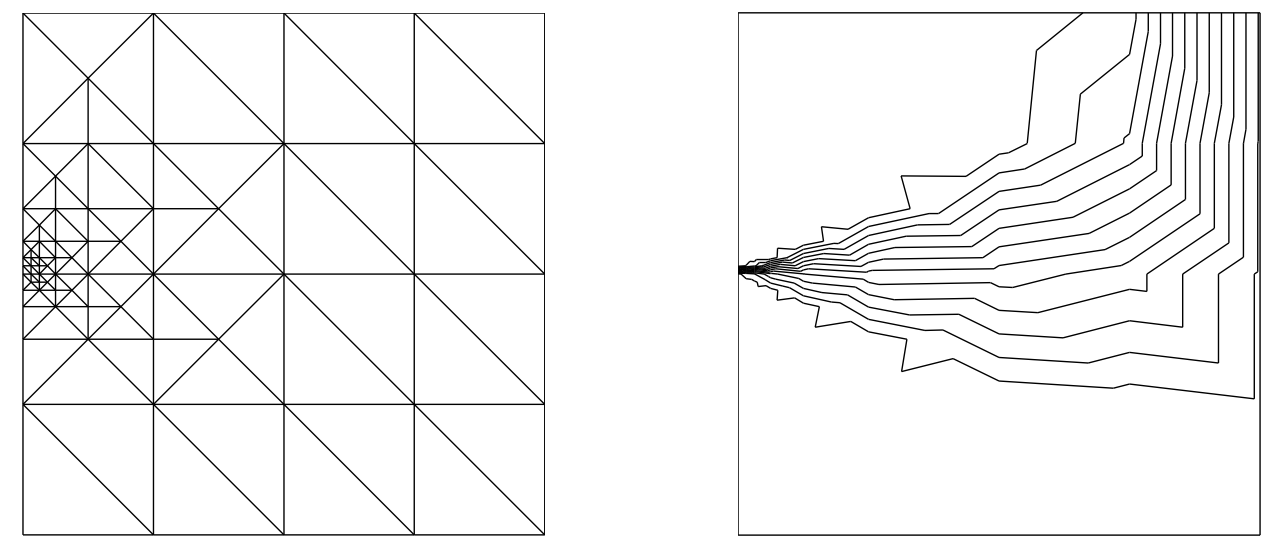

Figure 6: Initial grid and solution to 4.2: 68 dof.
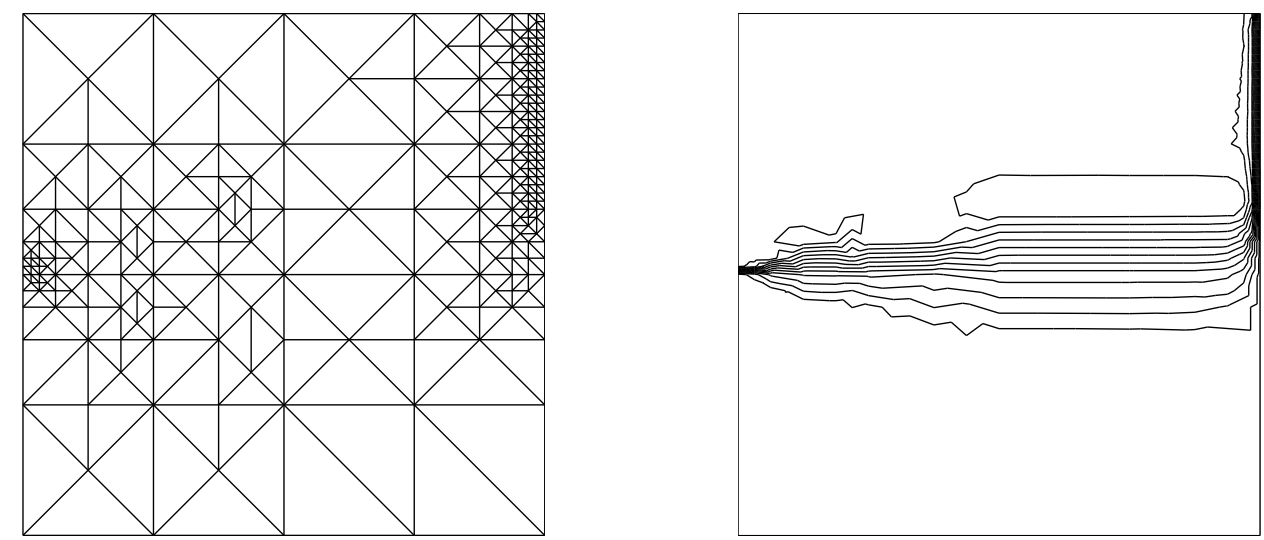

Figure 7: Adapted grid and solution to 4.2: 248 dof.

The computed solution and grid after 4 steps of adaptive refinement with subdivision parameter $\alpha=1 / 2$ is plotted in figure 7. Almost all of the initial refinement is concentrated in the boundary layer since the local error is dominated by the interior residual term. In the light of our analytic results (and the results in $\S 3$ ) we know that that the boundary layer refinement process will continue until $h_{T} / \epsilon$ is close to unity, at which point the estimator will be an effective measure of the local error so that the flux-jump term in (2.7) will become significant and force more refinement of the interior layer. This is illustrated in figure 8, where we plot the solution and grid after a further 10 steps 
of the adaptive refinement process. Although an oscillatory pattern to the refinement seems to develop, it is quite evident that our simple error estimator provides an effective refinement indicator even in the presence of boundary layers.
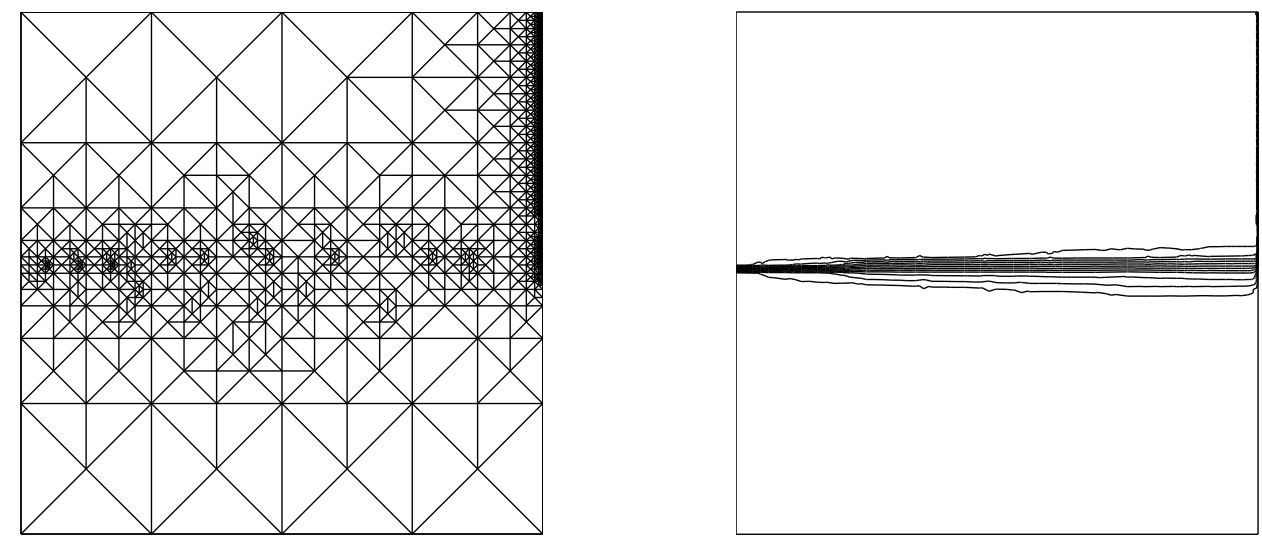

Figure 8: Adapted grid and solution to 4.2: 2472 dof

\section{$5 \quad$ Summary}

Our theoretical and numerical results suggest that our simple error estimator based on the solution on local Poisson problems provides an effective refinement indicator for convection-diffusion problems as long as streamline-diffusion stabilisation is built in to the methodology.

This leads to a coherent framework for solving the linearised Navier-stokes equations, since we know that effective estimators for incompressible Stokes equations can be computed by solving independent local Poison problems for each velocity component, see Kay \& Silvester [12]. We anticipate that a combination of the approach discussed herein together with that in [12] will provide the basis for a cheap and effective error estimator for incompressible flow problems.

\section{References}

[1] A. N. Agarwal and P.M. Pinsky, 1996. Stabilized element residual method (SERM): A posteriori error estimation for the advection-diffusion equation, $J$. Comp. Appl. Math., 74 pp. 3-17. 
[2] M. Ainsworth, 1994. The performance of Bank-Weiser's error estimator for quadrilateral elements, Num. Meth. PDEs., 10 pp. 609-623.

[3] M. Ainsworth And I. BabušKa, 1999. Reliable and robust a posteriori error estimation for singularly perturbed reaction-diffusion problems, SIAM J. Numer. Anal., 36 pp. 331-353.

[4] M. Ainsworth and J. T. Oden, 1992. A procedure for a posteriori error estimation for $h-p$ finite element methods, Comp. Meth. Appl. Mech. Eng., 101 pp. 73-96.

[5] I. BabušKa, T. Strouboulis, C.S. Upadhyay and S.K. Gangaraj, 1994. A model study of the quality of a posteriori error estimators for linear elliptic problems error estimation in the interior of patchwise uniform grids of triangles, Comp. Meth. Appl. Mech. Eng., 114 pp. 307-378.

[6] R. E. BAnk And A. Weiser, 1985. Some a posteriori error estimators for elliptic partial differential equations, Math. Comp., 44 pp. 283-301.

[7] D. Braess, 1997. Finite Elements, Cambridge University Press.

[8] P. ClÉment 1975. Approximation by finite element functions using local regularization, R.A.I.R.O. Anal. Numér., 2 pp. 77-84.

[9] B. Fischer, A. Ramage, D. J. Silvester and A. J. Wathen, 1999. On parameter choice and iterative convergence for stabilised discretisations of advectiondiffusion problems, Comp. Meth. Appl. Mech. Eng. 179 pp. 179-196.

[10] P.M. GResho And R.L. SAni, 1998. Incompressible Flow and the Finite Element Method, John Wiley.

[11] C. Johnson, U. NÄvert And J Pitkäranta 1984. Finite element methods for linear hyperbolic problems, Comp. Meth. Appl. Mech. Eng., 45 pp. 285-312.

[12] D. Kay And D. Silvester, 2000. A posteriori error estimation for stabilised mixed approximations of the Stokes equations, SIAM J. Sci. Comput. 21 pp. 1321-1336.

[13] H. G. Roos, M. Stynes And L. Tobiska, 1996. Numerical Methods for Singularly Perturbed Differential Equations, Springer-Verlag. 
[14] R. Verfürth, 1998. A posteriori error estimators for convection diffusion equations, Numer. Math., 80 pp. 641-663.

[15] R. Verfürth, 1996. A Review of A Posteriori Error Estimation and Adaptive Mesh-Refinement Techniques, Wiley-Teubner. 